OPEN ACCESS

Edited by:

Moriya Tsuji,

Columbia University Irving Medical

Center, United States

Reviewed by:

John Pak,

Chan Zuckerberg Biohub,

United States

Thorsten Demberg,

Baylor College of Medicine,

United States

*Correspondence:

Deborah H. Fuller

fullerdh@uw.edu

Specialty section:

This article was submitted to Vaccines and Molecular Therapeutics,

a section of the journal

Frontiers in Immunology

Received: 23 October 2021 Accepted: 01 December 2021 Published: 21 December 2021

Citation:

O'Connor MA, Erasmus JH, Randall $S$,

Archer J, Lewis TB, Brown B,

Fredericks M, Groenier S, Iwayama N,

Ahrens C, Garrison W, Wangari S,

Guerriero KA and Fuller DH (2021) A

Single Dose SARS-CoV-2 Replicon

RNA Vaccine Induces Cellular and

Humoral Immune Responses in Simian

Immunodeficiency Virus Infected and

Uninfected Pigtail Macaques.

Front. Immunol. 12:800723.

doi: 10.3389/fimmu.2021.800723

\section{A Single Dose SARS-CoV-2 Replicon RNA Vaccine Induces Cellular and Humoral Immune Responses in Simian Immunodeficiency Virus Infected and Uninfected Pigtail Macaques}

\author{
Megan A. O'Connor ${ }^{1,2}$, Jesse H. Erasmus ${ }^{1,3}$, Samantha Randall ${ }^{1}$, Jacob Archer ${ }^{1,3}$, \\ Thomas B. Lewis ${ }^{1,2}$, Brieann Brown ${ }^{1,2}$, Megan Fredericks ${ }^{1,2}$, Skyler Groenier ${ }^{1}$, \\ Naoto Iwayama ${ }^{2}$, Chul Ahrens ${ }^{2}$, William Garrison ${ }^{2}$, Solomon Wangari ${ }^{2}$, \\ Kathryn A. Guerriero ${ }^{2}$ and Deborah H. Fuller ${ }^{1,2 *}$

\begin{abstract}
1 Department of Microbiology, University of Washington, Seattle, WA, United States, ${ }^{2}$ Washington National Primate Research Center, University of Washington, Seattle, WA, United States, ${ }^{3}$ HDT Bio, Seattle, WA, United States
\end{abstract}

The ongoing COVID-19 vaccine rollout is critical for reducing SARS-CoV-2 infections, hospitalizations, and deaths worldwide. Unfortunately, massive disparities exist in getting vaccines to vulnerable populations, including people living with HIV. Preliminary studies indicate that COVID-19 mRNA vaccines are safe and immunogenic in people living with HIV that are virally suppressed with potent antiretroviral therapy but may be less efficacious in immunocompromised individuals. This raises the concern that COVID-19 vaccines may be less effective in resource poor settings with limited access to antiretroviral therapy. Here, we evaluated the immunogenicity of a single dose COVID-19 replicon RNA vaccine expressing Spike protein (A.1) from SARS-CoV-2 (repRNA-CoV2S) in immunocompromised, SIV infected and immune competent, naïve pigtail macaques. Moderate vaccine-specific cellular Th1 T-cell responses and binding and neutralizing antibodies were induced by repRNA-CoV2S in SIV infected animals and naïve animals. Furthermore, vaccine immunogenicity was elicited even among the animals with the highest SIV viral burden or lowest peripheral CD4 counts prior to immunization. This study provides evidence that a SARS-CoV-2 repRNA vaccine could be employed to induce strong immunity against COVID-19 in HIV infected and other immunocompromised individuals.

Keywords: vaccine, nonhuman primate, SIV, COVID-19 vaccine, replicon RNA, SARS-CoV-2

\section{INTRODUCTION}

Global implementation of vaccines against COVID-19 are necessary to reduce SARS-CoV-2 infections, hospitalizations, and mortality. However COVID-19 vaccine immunogenicity can be lower in immunocompromised individuals $(1,2)$. Preliminary studies indicate that COVID-19 vaccination is safe and immunogenic in people living with HIV that are virally suppressed on potent 
combination antiretroviral therapy (cART) but may be less immunogenic in HIV-infected individuals that have limited access to cART and whom have low CD4 counts $(<350$ cell/ $\mathrm{mm}^{3}$ ) or are immunosuppressed (3-6). Access to cART that is needed to reduce HIV viral burden is limited in resource poor settings and hence untreated HIV infection is a driver of morbidity and mortality in people living with HIV. Furthermore, inequitable distribution of COVID-19 vaccines, especially in low- and middle-income countries, where HIV is endemic, prevents vaccine access by many vulnerable populations. The emergence of SARS-CoV-2 lineages, especially in countries with poor access to COVID-19 vaccines, further jeopardizes achieving complete vaccine coverage. Thus, from a public health perspective it is necessary to study COVID19 disease and vaccines in immunosuppressed and untreated HIV populations.

Recent reports demonstrate that HIV infection is an independent risk factor for severe COVID-19 and mortality (7). Additionally, people living with HIV have a disproportionate risk of cardiovascular, kidney, and liver disease, cancer, and diabetes, which are co-morbidities associated with increased risk of COVID-19-related hospitalization and death (8-10). Many of the studies evaluating COVID-19 risk in people living with HIV do not capture the $>25 \%$ of individuals who are untreated (11) and studies are needed to understand specific risks during untreated or advanced HIV.

Previously, we reported that vaccination with a single dose or two doses of a self-amplifying replicon RNA (repRNA) SARS-CoV-2 vaccine, encoding the SARS-CoV-2 (S) spike protein, repRNA-CoV2S, generated robust binding and neutralizing antibody $(\mathrm{nAb})$ responses in mice and nonhuman primates (NHP) (12). A phase I human clinical trial in India with this vaccine is nearing completion and phase I trials in Brazil, Korea, Philippines, China, and the US and a phase II/III trial in India are pending (13-15). Decreased vaccine immunogenicity to infectious diseases remains a significant issue for people living with HIV, even among those with access to potent cART (16). To overcome these limitations, people living with HIV often require higher vaccine doses, additional booster doses or co-delivery with potent adjuvants to develop sufficient levels of immunity (17). Current COVID-19 mRNA vaccines have $>90 \%$ efficacy at preventing COVID-19 and hospitalization, however in individuals who are immunocompromised, vaccine efficacy is reported to be $\sim 20-30 \%$ lower (18-20). Unlike "conventional" mRNA vaccines, repRNA vaccines trigger innate pathways, activate Toll-like receptors and promote cross-priming that can contribute to eliciting more robust immune responses (21). Consequently, this vaccine platform is self-adjuvating and ideal for inducing immune responses in immunosuppressed individuals. Here, we used the pre-clinical simian immunodeficiency virus (SIV) NHP model for human HIV infection to investigate repRNA-CoV2S vaccine immunogenicity in SIV-infected pigtail macaques during analytic antiretroviral treatment interruption.

\section{MATERIALS AND METHODS}

\section{Pigtail Macaques and Sample Collection}

A total of 15 male pigtail macaques (aged 2.1-5.1 years, 3.6-9.6 $\mathrm{kg}$ ) were used. Supplemental Table 1 details animal characteristics, including MHC haplotypes. Nine animals were experimentally infected with SIV (described in detail below) and 6 animals remained naïve. All animals received a single immunization ( 5 or $25 \mu \mathrm{g}$ ) of repRNA-CoV2S and were then studied for 6 weeks. As previously described (12), all animals were housed at the Washington National Primate Research Center (WaNPRC), an accredited facility the American Association for the Accreditation of Laboratory Animal Care International (AAALAC). All animal procedures were approved by the University of Washington's Institutional Animal Care and Use Committee (IACUC) (IACUC \#4266-14). Animals were sedated with an intramuscular injection $(10 \mathrm{mg} / \mathrm{kg})$ of ketamine (Ketaset ${ }^{\circledR}$; Henry Schein) for blood collections. Serum, plasma, and peripheral blood mononuclear cells (PBMCs) were isolated from whole blood as previously described (12). The animal's general health was observed daily and full physical exams were conducted at each experimental timepoint, as previously described (12). Only SIV-infected animals were euthanized at the study endpoint at 6 weeks post-immunization under deep anesthesia, in accordance with the 2007 American Veterinary Medical Association Guidelines on Euthanasia, by administration of Euthasol ${ }^{\circledR}$ (Virbac Corp., Houston, TX).

\section{SIV Infection and cART Regimen}

Nine pigtail macaques were infected intravenously with 10,000 infectious units (I.U.) of SIVmac239M (22) (gift from Dr. Brandon Keele, AIDS and Cancer Virus Program, Frederick National Laboratory for Cancer Research). Animals were given a combination antiretroviral therapy (cART) regimen for 26 weeks starting at 4 weeks post-SIV infection consisting of $1 \mathrm{~mL} /$ $\mathrm{kg}$ of a mixture of the following antiretrovirals administered daily by intramuscular (i.m.) injection: $2.5 \mathrm{mg} / \mathrm{kg}$ Dolutegravir (DTG) (Gilead Sciences, Foster City, CA), $5.1 \mathrm{mg} / \mathrm{kg}$ tenofovir disoproxil fumarate (TDF) (ViiV Healthcare, Research Triangle, $\mathrm{NC}$ ), and $30 \mathrm{mg} / \mathrm{kg}$ emtricitabine (FTC) (Gilead Sciences, Foster City, CA) in 15\% Kleptose in water (Roquette, Geneva, IL). These SIV-infected animals were previously enrolled in another study where following SIV infection and during CART, they received 3 doses of an experimental hepatitis $B$ virus (HBV) vaccine consisting of a combination of CD180 targeted DNA and recombinant protein vaccines comprised of $\mathrm{HBV}$ core and surface antigens $(n=4)(23)$ or a commercial Engerix-B vaccine (GlaxoSmithKline, Research Triangle Park, NC) $(n=5)$ (Supplemental Table 1). Analytic treatment interruption (ATI) was performed and then in response to the COVID-19 pandemic, these animals were re-enrolled in this study after viral loads had rebounded and were immunized with the repRNACoV2S vaccine at 8 weeks post-ATI. Animals were then euthanized 6 weeks after the single repRNA-CoV2S dose consistent with the original experimental endpoint associated 
with the HBV vaccine study. Animals receiving each $\mathrm{HBV}$ vaccine were evenly distributed between the repRNA-CoV2S vaccine groups (Supplemental Table 1).

\section{RepRNA-CoV2S Immunization}

Macaques were immunized with an alphavirus-derived replicon RNA SARS-CoV-2 vaccine encoding the full-length A.1 lineage (GenBank: MN908947.3) SARS-CoV-2 Spike (S) protein (repRNA-CoV2S). This repRNA vaccine is comprised of codon optimized DNA gene sequences fused to a c-terminal v5 epitope tag and cloned into a plasmid vector encoding untranslated regions and a non-structural open reading frame of Venezuelan equine encephalitis virus strain TC-83 (12). To protect repRNA from degradation and enhance stability, repRNA was coformulated with a Lipid InOrganic Nanoparticle (LION) emulsion consisting of inorganic superparamagnetic iron oxide (SPIO) nanoparticles and squalene core (HDT Bio, Seattle, WA). The stability, safety, and immunogenicity of repRNA-CoV2S was described previously in detail (12). The vaccine was prepared as previously described (12) and delivered by i.m. injection into the quadriceps and deltoid muscles. Animals received one of the following repRNA-CoV2S vaccine doses: 1) $25 \mu$ g delivered over 5 i.m. sites ( $\mathrm{n}=3 \mathrm{SIV}+, \mathrm{n}=3$ naïve), 2) $5 \mu$ g delivered over 5 i.m. sites ( $\mathrm{n}=3$ SIV+, $\mathrm{n}=3$ naïve), or 3 ) $5 \mu$ g delivered over 1 i.m. site [single site (SS)] $(n=3, S I V+)$, as shown in Supplemental Table 1. All injection sites were monitored post-immunization for any signs of local reactogenicity.

\section{Simian AIDS Measurements}

Animals were monitored for Simian AIDS as defined by the WaNPRC guidelines (24). Briefly, weights, complete blood counts, and peripheral blood CD4 counts were monitored at each specimen collection timepoint. SIV plasma viremia was evaluated by quantitative real time reverse transcription polymerase chain reaction (RT-PCR) by the Virology and Immunology Core at the WaNPRC, as previously described (24). Peripheral CD4 counts were determined from complete blood counts (12) (University of Washington Department of Laboratory Medicine) using flow cytometry based methods by the Virology and Immunology Core at the WaNPRC, as previously described (25).

\section{IL-4/IFN- $\gamma$ Enzyme-Linked Immunospot Assay (ELISPOT)}

Antigen-specific PBMCs secreting IL-4 or IFN- $\gamma$ were detected using a Human IFN- $\gamma /$ IL- 4 Double-Color ELISPOT (ImmunoSpot, Shaker Heights, Cleveland, OH), per manufacturer's protocol. Briefly, cryopreserved PBMC cells were thawed, and 1-3 x $10^{5}$ cells were stimulated for 48 hours with 11 SARS-CoV-2 Spike peptide pools (17- or 18-mers with 11 amino acid overlap) (Genscript, Piscataway, NJ) at a concentration of $1 \mu \mathrm{g} /$ $\mathrm{mL}$ per peptide. DMSO and Concanavalin A (ThermoFisher, Waltham, MA), were used as negative and positive controls, respectively, as previously described (12). Spots were counted on an Immunospot Analyzer with CTL Immunospot Profession Software (Cellular Technology Ltd., Shaker Heights, Cleveland, $\mathrm{OH}$ ). Spot forming cells (SFC) in peptide stimulated wells were computed following subtraction of SFCs detected in DMSO stimulated control wells and were considered positive if the number of SFC was $>1$ spot per $1 \times 10^{5}$ plated cells.

\section{SARS-CoV-2 Binding Antibody}

Antigen-specific IgG responses were detected in the sera by enzyme linked immunosorbent assay (ELISA) using a recombinant SARSCoV-2 S protein as the capture (26), and performed as previously described (12). Briefly, ELISA plates were coated with $1 \mu \mathrm{g} / \mathrm{mL}$ antigen and serially diluted serum samples were added and detected via anti-monkey IgG-HRP (Southern Biotech, Birmingham, AL) and then developed using a TMB substrate (Fisher Scientific, Waltham, MA). Plates were analyzed at 405nm (ELX808, Bio-Tek Instruments Inc) and serum concentrations were determined from a standard curve, as previously described (12).

\section{SARS-CoV-2 Neutralizing Antibody}

Eighty percent (80\%) plaque-reduction neutralizing antibody titers (PRNT80) against the SARS-CoV2/WA/2020 (BEI Resources, Manassas, VA) were determined in two-fold serial diluted heat inactivated serum, as previously described (12). Briefly, serum and virus were incubated and plated onto Vero E6-TMPRSS2 cells (gift from Dr. Michael Gale, University of Washington) and incubated for $1 \mathrm{hr}$ at $37^{\circ} \mathrm{C}$. Following adsorption, wells were overlaid with $0.2 \%$ agarose in DMEM and incubated for 2 days at $37^{\circ} \mathrm{C}$. The overlay was then removed, washed, fixed using 10\% formaldehyde (Sigma-Aldrich, St. Louis, $\mathrm{MO}$ ), and stained with $1 \%$ crystal violet (Sigma-Aldrich, St. Louis, MO) in 20\% EtOH (Fisher Scientific, Waltham, MA). Plaques were enumerated and percent neutralization was calculated relative to the virus-only control.

\section{Quantification of Gut Barrier Dysfunction}

Plasma quantification by ELISA of human soluble CD14 (sCD14), human $\mathrm{C}$-reactive protein (CRP), human fatty acid binding protein 2 (FABP2) (Fisher Scientific, Waltham, MA) or human LPS binding protein (LBP) (Biometec, Germany) was performed per the manufacturer's instruction. Plasma was diluted as follows: 1:200 (sCD14), 1:2 (FABP2), 1:1,000 (CRP), or 1:3 (LBP). Results were analyzed using a four-parameter logistic (4-PL) function for fitting standard curves using Prism version 8.4.3 (GraphPad).

\section{Statistical Analyses}

Statistical analyses were conducted in Prism v.8.4.3 (Graphpad, San Diego, CA, USA). Comparisons between groups were determined by Mann-Whitney test. Correlations were evaluated by Spearman Rank test. Statistical significance was considered achieved when the $p$-values were $<0.05$.

\section{RESULTS}

\section{Experimental Design of repRNA-CoV2S Vaccination in Naïve and Immunocompromised, SIV-Infected Pigtail Macaques}

Previously, we demonstrated robust immunogenicity with one or two doses of the repRNA-CoV2S vaccine in naïve pigtail macaques 
(PTM) (Macaca nemestrina) (12). Given the ability of repRNA vaccines to induce robust innate immune responses (27), we hypothesized that repRNA-CoV2S would also be able to induce strong antibody and $\mathrm{T}$ cell responses in immunocompromised SIVinfected NHPs. To determine if this vaccine could induce strong immune responses in a non-human primate model of HIV/AIDS that closely resembles immunocompromised people living with HIV, we immunized SIV infected PTMs (N=9) 8 weeks after cART was withdrawn and when viral loads had rebounded. The PTMs were previously infected intravenously with SIVmac239M, a barcoded virus allowing for the distinction of almost 10,000 isogenic clones (22), and then put on cART for a period of 6 months starting 4 weeks after infection (Figure 1A). During the cART period, and as part of another experiment, the PTMs received an experimental hepatitis B virus (HBV) vaccine (Supplemental Table 1) and then underwent an analytic treatment interruption (ATI) that resulted in rapid rebound of SIV viral replication.

Vaccine immunogenicity in the nine SIV infected PTMs was compared to six naïve PTMs given the same repRNACoV2S vaccine. Previously we evaluated administration of repRNA-CoV2S at $50 \mu \mathrm{g}$ over one injection site or $250 \mu \mathrm{g}$ over five injection sites (12). To support our clinical development activities with the repRNA-CoVS vaccine platform, we employed a dose de-escalation study exploring the immunogenicity at two lower vaccine doses $(25 \mu \mathrm{g}$ or $5 \mu \mathrm{g})$ in naïve PTMS. Here, we did parallel vaccine immunogenicity studies in SIV-infected pigtail macaques at these lower doses. To continue to bridge our preclinical vaccine model for use in the clinic, we also tested administration of the $5 \mu \mathrm{g}$ vaccine in the SIV+ cohort delivered over five $(n=3)$ or a single (SS, $n=3)$ injection site(s) (Supplemental Table 1). Altogether, the vaccinated experimental groups were as follows ( $n=3$ /group): $\mathrm{SIV}+25 \mu \mathrm{g}$, $\mathrm{SIV}+5 \mu \mathrm{g}, \mathrm{SIV}+5 \mu \mathrm{g}$ SS, naïve $25 \mu \mathrm{g}$, naïve $5 \mu \mathrm{g}$ (Figure $\mathbf{1 A}$ and Supplemental Table 1). All SIV-infected animals were immunized with a single dose of the repRNA-CoV2S vaccine at 8 weeks after cART withdrawal. At the time of immunization, the median \pm interquartile range level of SIV viral RNA in the plasma was $3.9 \pm 4.6 \log _{10}$ copies $/ \mathrm{mL}$, with a single animal (Z15182, SIV+ $5 \mu \mathrm{g}$ ) exhibiting SIV viral control at or below the limit of detection (Figure 1B and Supplemental Table 2). In addition, frequencies of peripheral blood CD4 counts ranged from 402 to 1046 cells/ $\mu \mathrm{L}$ of blood (Supplemental Table 1) and corresponded to declines of up to $75.3 \%$ relative to baseline levels measured prior to SIV infection (Figures 1C, D and Supplemental Table 1). A single animal (Z14302, SIV+ $5 \mu \mathrm{g})$ did not have depletion of peripheral CD4 T-cells at the time of vaccination (Figure 1D and Supplemental Table 1). Evidence of gut barrier dysfunction was minimal at the time of vaccination, with only 2 animals displaying elevated plasma levels of lipopolysaccharide binding protein (LBP) compared to pre-infection levels (Supplemental Figure 2). Thus, at 8 weeks post-cART withdrawal when the vaccine was administered, 8/9 SIV-infected animals exhibited viral rebound and $8 / 9$ exhibited a range in immunosuppression as indicated by declines in peripheral CD4 T cell counts. Animals were monitored for 6 weeks, and blood was collected prior to vaccination and at 14, 28 , and 42 or 44 days post immunization (DPI) to evaluate vaccine
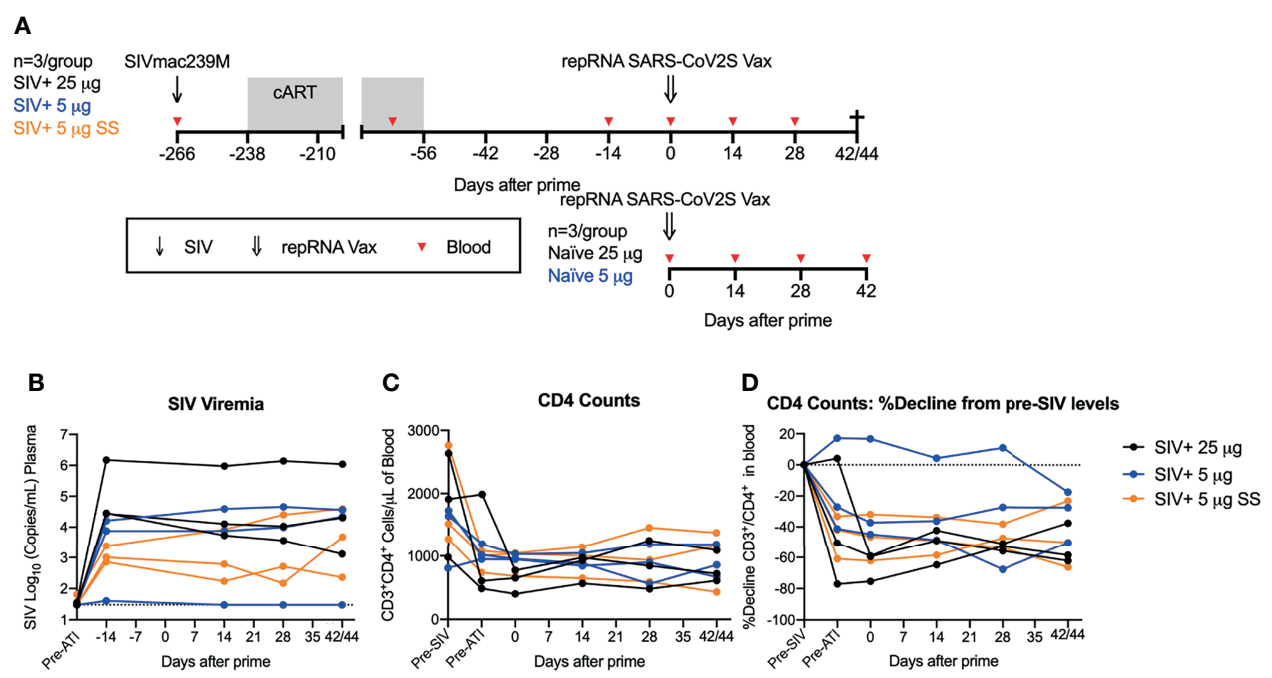

FIGURE 1 | repRNA-CoV2S vaccination in SIV-infected, immunosuppressed pigtail macaques. (A) Pigtail macaques $(n=9)$ were infected with SIVmac239M and put on 26 weeks of CART starting 4 weeks after infection. During cART animals received an HBV DNA and protein vaccine regimen, comprised of HBV core and surface antigens and anti-CD180, prior to enrollment in the COVID-19 vaccine study. CART was stopped 8 weeks prior to receiving an intramuscular immunization of repRNA-CoV2S (RepRNA vax) $(25 \mu \mathrm{g}$ ( $\mathrm{n}=3$, black circles); $5 \mu \mathrm{g}$ [ $\mathrm{n}=6$ (blue and orange circles)] delivered over 5 sites (black and blue circles) or single site (SS) (orange circles). repRNA-CoV2S was also delivered to naïve control animals [25 $\mu \mathrm{g}(\mathrm{n}=3) ; 5 \mu \mathrm{g}(\mathrm{n}=3)]$. Blood was collected at baseline and days 10, 14, 28, and 42/ $44 \mathrm{DPI}$. SIV+ animals underwent an experimental necropsy associated with the HBV-vaccine protocol on days $42 / 44$ post-vaccination. (B) Plasma levels of SIV viral RNA levels were measured by RT-PCR, the dotted line the limit of detection (30 copies $/ \mathrm{mL}$ of plasma). (C) Peripheral blood CD3+CD4+ T-cell counts were quantified from the complete blood count (CBC) following flow cytometry analysis. (D) Decline in peripheral blood CD3+CD4+ T-cell counts were calculated relative to the percent of pre-SIV infection levels. The dotted line indicates pre-SIV infection levels. 
safety and immunogenicity including analysis of cellular and humoral immune responses. Vaccine follow-up of 6 weeks (42/ 44 days) in the SIV-infected animals was limited by an experimental endpoint associated with the original $\mathrm{HBV}$ vaccine study (see Methods). Consistent with our previous study in uninfected animals (12), no acute adverse reactions were observed in any of the animals following immunization (Supplemental Figure 1).

\section{Low Doses of repRNA-CoV2S Induce Strong Antibody and Modest T Cell Responses in SIV-Infected Pigtail Macaques}

Cellular and humoral immune responses to the vaccine were evaluated at 28 and 42/44 DPI. Within the SIV cohort, similar levels of antibody and $\mathrm{T}$ cell responses were observed for both the 5 and 25 ug dose levels regardless of whether the dose was administered over a single or five sites; therefore, data were combined for comparative analyses (Supplemental Figure 3 and Supplemental Table 2). In our previous studies, we measured IFN- $\gamma \mathrm{T}$ cell responses by ELISPOT and polyfunctional $\mathrm{T}$ cell responses expressing IFN- $\gamma$, IL-2, IL-4, IL-17A, TNF- $\alpha$, MIP-1 $\beta$, and/or Granzyme B/CD107a by intracellular cytokine staining (ICS). Only mild/moderate Tcell responses elicited by repRNA-CoV2S were detected and T cell responses were almost exclusively IFN- $\gamma$ secreting cells (12). Therefore, in this study, we focused on IFN- $\gamma$ and IL- 4 T cell responses measured by ELISPOT in our analysis to investigate the effects of SIV infection on Th1 and Th2 responses. Modest frequencies of IFN- $\gamma$ (Th1) producing T-cells were induced after a single dose in SIV-infected and naïve PTMs (Figure 2A). In addition, the magnitude of the IFN- $\gamma$ T-cell response in the SIV-infected macaques were consistent with levels we previously observed following immunization of naïve PTMs with higher doses (50-250 $\mu \mathrm{g}$ ) of the same vaccine (12). Overall, T-cell responses were detected in $30-45 \%$ of all animals (4/9 SIV+ and 2/6 naïve) at 42/44 DPI. Vaccine induced Th2 (IL-4) T-cells were detected at very low levels only in a single SIV-infected animal at 28 DPI and in none of the naïve animals (Supplemental Figure 3). The Th1 T-cell response was primarily directed against the Spike 2 subunit (Supplemental Figure 4) in both SIV+ and naïve animals with only 2 PTM (1 SIV+ and 1 naïve) exhibiting a dominant T-cell response against the Spike 1 subunit that contains the receptor binding domain (RBD). These results are in contrast to our previous study where we showed that 2 to 10 -fold higher doses $(50-250 \mu \mathrm{g})$ of the repRNA-CoV2S vaccine in naïve PTMs induced a dominant $\mathrm{T}$ cell response against the $S 1 / \mathrm{RBD}$ region (12). These data suggest that the dose of repRNA-CoV2S may influence the specificity or repertoire of $\mathrm{T}$-cell responses.

We next evaluated humoral responses in the sera. Robust binding antibodies (bAb) against the $\mathrm{S}$ protein were detected in all animals (Figure 2B). Concentrations of anti-Spike IgG of 0.35 to $28 \mu \mathrm{g} / \mathrm{mL}$ observed at day 28 in this study were comparable to IgG concentrations ( 0.5 to $45 \mu \mathrm{g} / \mathrm{mL}$ ) previously reported after a single, higher dose of 50 or $250 \mu \mathrm{g}$ repRNA-CoV2S (12). Despite seroconversion in all PTM, variable levels of antibody virus neutralizing titers were detected 28-42/44 DPI (Figure 2C). At 28 DPI, $1 / 9$ SIV-infected and 2/6 naïve animals had detectable neutralizing antibody (nAb) responses and by $42 / 44 \mathrm{DPI}, \mathrm{nAb}$ were detected in 4/9 and 2/6 in SIV-infected and naïve PTM, respectively (Figure 2C). At $42 / 44 \mathrm{DPI}, \mathrm{nAb}$ responses were detected in $66 \%(4 / 6)$ of PTMs receiving the $25 \mu \mathrm{g}$ dose versus $22 \%$ (2/9) of PTMs receiving the $5 \mu \mathrm{g}$ dose, irrespective of SIV status (Figure 2C). Overall, a better responder rate and significantly higher levels of $\mathrm{nAb}$ responses were detected at the $25 \mu \mathrm{g}$ versus the $5 \mu \mathrm{g}$ dose (Supplementary Table 2). Furthermore, nAb levels induced with the $25 \mu \mathrm{g}$ dose in both naïve and SIV-infected PTMs were comparable with levels induced using higher doses of the vaccine in our previous study in naïve PTMs (12), but the response rate was lower in all animals receiving doses $<25 \mu \mathrm{g}$. These data indicate that the dose of repRNA-SARS-CoV2S needed to induce optimum nAb responses against SARS-CoV-2 is $>5 \mu \mathrm{g}$.
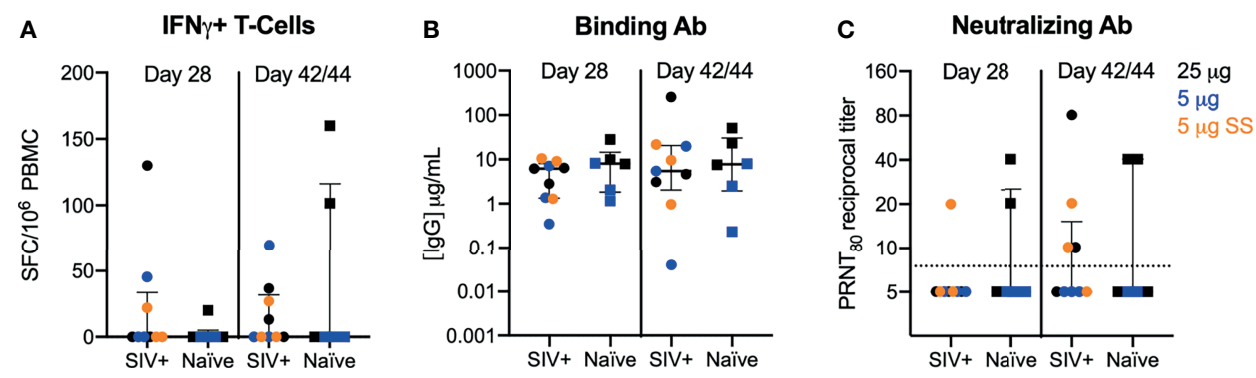

FIGURE 2 | repRNA-CoV2S vaccination in SIV-infected pigtail macaques induces robust cellular and humoral immune responses. PBMCs and sera were isolated from blood at 28 and 42/44 DPI for vaccine immunogenicity. (B) The magnitude of IFN- $\gamma$-producing T-cells were measured in PBMCs following 48-hour stimulation with 11 peptide pools encompassing the SARS-CoV-2 spike (S) protein using a dual IFN- $\gamma /$ IL-4 ELISpot assay. The number of spot forming cells (SFCs) per $10^{6}$ PBMC are shown. IL-4-producing T cell responses were low or undetectable in all animals post-vaccination and are not shown (see Supplementary Figure 4). (C) Serum anti-S IgG enzyme linked immunosorbent assays were measured by ELISA and (D) 80\% plaque-reduction neutralizing antibody titers (PRNT80) against the SARS-CoV2NA/2020 isolate were measured by plaque reduction neutralization test (B-D) Medians with interquartile ranges are shown. No significance was found by Mann-Whitney test between SIV+ and naïve animals at either timepoint. 
The Immunogenicity of the repRNA-CoV2S Vaccine Is Not Impacted by the Level of SIV-Induced Immunosuppression

At the time of immunization, the SIV-infected macaques exhibited varying levels of SIV disease progression, with pre-vaccination viral loads ranging from 1.61 to $6.17 \log _{10}$ SIV RNA copies/mL and CD4 counts ranging from 402 to $>1000$ cells $/ \mu \mathrm{L}$ of blood (Supplemental Table 1). To determine if the immunogenicity of the repRNA-CoV2S vaccine was impacted by SIV disease phenotypes, $\mathrm{bAb}, \mathrm{nAb}$ and frequencies of Th1 T-cell responses measured 42/44 days post-vaccination were compared to viral loads and CD4 counts present just prior to vaccination. Overall, there was no significant correlation between the magnitude of $\mathrm{bAb}, \mathrm{nAb}$ or $\mathrm{T}$ cell responses and viral loads, frequencies of peripheral CD4 counts (Figure 3), relative decline in CD4 counts compared to pre-SIV baseline levels. (Supplemental Figure 5), nor with plasma levels of markers of gut barrier dysfunction (Supplemental Figure 6). Collectively, these data demonstrate that the immunogenicity of the repRNA-CoV2S was not impacted by SIV-induced immunosuppression.

\section{DISCUSSION}

Here, we demonstrate a SARS-CoV-2 alphavirus-derived replicon RNA vaccine formulated with a novel Lipid InOrganic Nanoparticle (LION) emulsion (repRNA-CoV2S) induced robust cellular and humoral immune responses after a single immunization in immunocompromised SIV-infected animals as a highly relevant model of untreated HIV infection in humans. Collectively, this pilot, proof-of-concept study provides strong evidence that a SARS-CoV-2 repRNA vaccine could be employed to induce immunity against COVID-19 in HIV infected and other immunocompromised individuals.

Preliminary studies indicate that mRNA or adenovirusvectored COVID-19 vaccination is safe and immunogenic in treated and virally suppressed people living with $\operatorname{HIV}(3,5,6)$. In addition, antibody and $\mathrm{T}$-cell responses following natural SARSCoV-2 infection in virally suppressed HIV infected individuals on antiretroviral drug therapy do not appear to be dampened when compared to HIV uninfected individuals (28). However, there is evidence that responsiveness to COVID-19 vaccination and the durability of vaccine-induced immunity may be dampened in non-HIV-associated immunocompromised individuals $(1,2,18,19)$ and individuals living with HIV who are non-virally suppressed and have low CD4 counts (3-5). In addition, there is growing evidence that there may be a role of $\mathrm{HIV}$-associated immune impairment, in particular lower $\mathrm{CD} 4{ }^{+}$ T-cell counts, with susceptibility to SARS-CoV-2 infection or COVID-19 severity $(29,30)$. Our data suggests that selfamplifying repRNA vaccines may enable induction of protective immunity in these vulnerable, immunocompromised populations. Importantly, unlike current mRNA COVID-19 vaccines, this vaccine platform utilizes an ad-mix formulation that can be manufactured independently of the RNA component and combined with the RNA vaccine immediately prior to immunization. The LION formulation used to deliver our

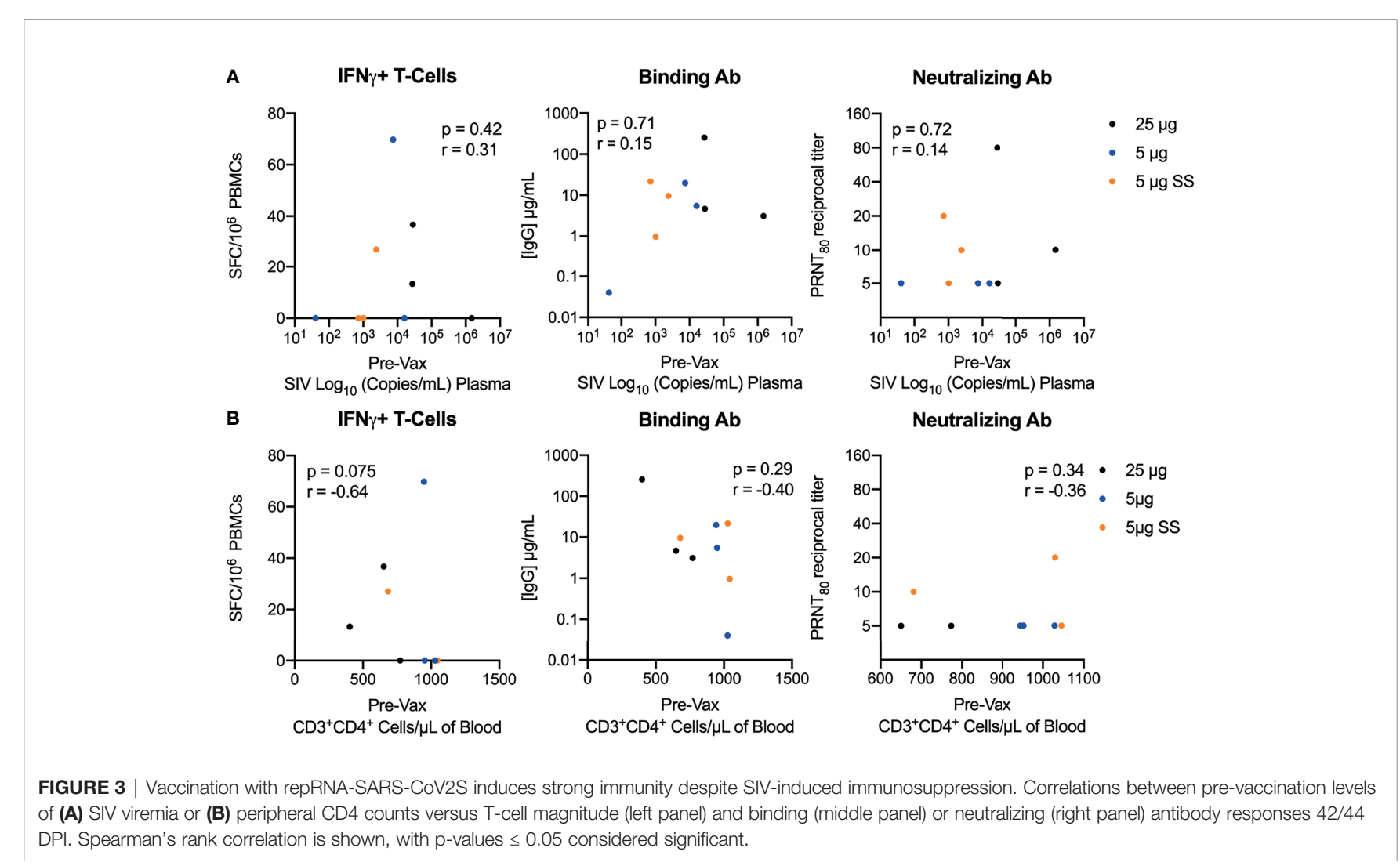


repRNA vaccine can be stored long-term at $4^{\circ} \mathrm{C}$ or room temperature (12). The stability of this vaccine platform makes it more amenable than currently licensed mRNA vaccines for rapid distribution in low income and resource poor settings where it can be locally stockpiled and readily available for coformulation with an updated RNA vaccine customized to each region depending on local virus epidemiology and in response to emerging variants of concern and future pandemics. Since the RNA vaccine does not need to be encapsulated into lipid nanoparticles under a regulated manufacturing process, formulating variant-specific vaccines with LION is more flexible and can be more rapidly customized.

Many nonhuman primate species including rhesus macaques (Macaca mulatta), cynomolgus macaques (Macaca fascicularis), African green monkeys (Chlorocebus aethiops) and pigtail macaques reported here (Macaca nemestrina), have been critical for investigating COVID-19 disease and pre-clinical testing of COVID-19 vaccines $(31,32)$. Recent findings from Melton et al., demonstrate moderate SARS-CoV-2 disease in pigtail macaques (33), as compared to the more mild disease observed in rhesus macaques, but with similar levels of viral load in respiratory mucosa between the two species (34). There are several unique features of the pigtail macaque model of HIV infection that are worth noting. SIV infection in pigtail macaques results in a more rapid progression to AIDS-defining events than rhesus macaques $(35,36)$. The increased disease progression in the pigtail is not due to higher levels of SIV replication, but rather is because of a higher propensity for decreased gut barrier function and increased immune activation $(35,37)$. Furthermore, studies have demonstrated the pigtail macaque as a model for studying cardiovascular disease and chronic obstructive pulmonary disease (COPD) $(38,39)$ and thus could be valuable for studying SARS-CoV-2 co-morbidities. The elderly are more vulnerable to severe COVID-19 and death and aged nonhuman primates are useful for recapitulating aspects of severe COVID-19, including acute respiratory distress syndrome (ARDS) and pneumonia (40, 41 ), but the scarcity of this resource limits its widespread use in COVID-19 research. Additional studies are needed to determine if repRNA-2S retains its immunogenicity in older naïve and SIVinfected macaques.

As a pilot, there are limitations to this study, including the short duration of post-immunization follow-up (6 weeks), the use of a prime only immunization, delivery of a subthreshold vaccine dose $(5 \mu \mathrm{g})$ that resulted in suboptimal immune responses even in naïve animals, and use of only male animals. Neutralizing antibody responses across the SIV and naïve groups were detected in $30-45 \%$ of animals after 6 weeks. This is in contrast to our previous results in animals given a 50 or $250 \mu \mathrm{g}$ dose (12), suggesting that a longer period of time or a booster immunization may be needed to generate maximum $\mathrm{nAb}$ responses at lower vaccine doses $(<25 \mu \mathrm{g})$. Despite these limitations, the ability of this vaccine to induce immune responses in fully immune competent uninfected and SIV +/CD4-depleted macaques provides evidence that the repRNACoV2S/LION vaccine delivery platform can induce immunity even in the context of immunosuppression. Further studies are needed to directly compare the repRNA/LION to a standard mRNA/lipid nanoparticle vaccine formulation to determine if the ability of the repRNA/LION vaccine to induce robust immune responses in SIV+ immunocompromised animals is unique.

Recent studies suggest $\mathrm{T}$-cells may not be required for generation of anamnestic clearance of SARS-CoV-2 reinfection (42). Thus, production of robust, long-lived humoral immunity following COVID-19 vaccination even in the setting of HIV-induced CD4 depletion and immune exhaustion may be possible. Vaccine seroprotection in people living with HIV can be shorter than in uninfected individuals (43), but there is evidence that durable humoral immunity is detected $>6$ months after SARS-CoV-2 infection irrespective of HIV status (44). It is possible that HIV/SIV-induced immune dysfunction, especially in individuals with untreated or advanced HIV (45), may negatively impact the durability of repRNA-CoV2S vaccine immune responses and further studies are needed to investigate this. We previously found that the repRNA vaccine platform drives a strong humoral response, but mild cellular immune responses in naïve macaques (12). The ability of the repRNA vaccine to induce robust antibody even in the presence of modest or low $\mathrm{T}$ cell responses may be beneficial in individuals with $\mathrm{T}$ cell deficiencies or immune dysfunction. In support of this possibility, the repRNA vaccine induced robust humoral immune responses in animals with low to moderate peripheral CD4+ T-cell counts of $400-750$ cells $/ \mu \mathrm{L}$ of blood, but further evaluation of COVID-19 vaccines in a setting of severe CD4 depletion $(<250$ cells $/ \mu \mathrm{L})$ is needed.

In addition, further studies are needed to determine the impact of HIV infection, in particular, T- and B-cell dysfunction, on the induction of neutralizing and binding $A b$ responses and on SARS-CoV-2 viral evolution and viral pathogenesis. In immunocompromised individuals, prolonged and persistent SARS-CoV-2 infection could provide fertile ground for viral evolution of more virulent strains $(46,47)$, that when transmitted could contribute to "breakthrough infections" in vaccinated individuals (45). In conclusion, the repRNA/LION vaccine platform is a viable next generation COVID-19 vaccine to induce protective levels of antibody responses in immunocompromised individuals and may be an ideal COVID-19 vaccine candidate for distribution in resource limited settings.

\section{DATA AVAILABILITY STATEMENT}

The raw data supporting the conclusions of this article will be made available by the authors, without undue reservation.

\section{ETHICS STATEMENT}

The animal study was reviewed and approved by University of Washington's Institutional Animal Care and Use Committee (IACUC) (IACUC \#4266-14). 


\section{AUTHOR CONTRIBUTIONS}

Conceptualization: MO, JE, and DF. Methodology: MO, JE, SR, JA, TL, BB, MF, and SG. Formal analysis: JE, SR, JA, TL, BB, MF, and SG. Writing-original draft preparation: $M O$ and DF. Writing-review and editing: MO'C, JE, SR, JA, TL, BB, MF, and DF. Animal studies and veterinary care: NI, CA, SW, WG, and KG. Visualization: MO. Supervision: MO, JE, and DF. Funding acquisition: JE and DF. All authors contributed to the article and approved the submitted version.

\section{FUNDING}

This work was supported by the National Institute of Health (NIH) grant numbers R56-AI141494 (DF/Clark), P51OD010425 (PI-Sullivan, DF Co-I, MO'C Co-I), NIH/NIAID Centers of Excellence for Influenza Research and Surveillance

\section{REFERENCES}

1. Herishanu Y, Avivi I, Aharon A, Shefer G, Levi S, Bronstein Y, et al. Efficacy of the BNT162b2 mRNA COVID-19 Vaccine in Patients With Chronic Lymphocytic Leukemia. Blood (2021) 137(23):3165-73. doi: 10.1182/blood.2021011568

2. Grupper A, Rabinowich L, Schwartz D, Schwartz IF, Ben-Yehoyada M, Shashar M, et al. Reduced Humoral Response to mRNA SARS-Cov-2 BNT162b2 Vaccine in Kidney Transplant Recipients Without Prior Exposure to the Virus. Am J Transplant (2021) 21(8):2908. doi: 10.1111/ajt.16615

3. Frater J, Ewer KJ, Ogbe A, Pace M, Adele S, Adland E, et al. Safety and Immunogenicity of the ChAdOx1 nCoV-19 (AZD1222) Vaccine Against SARS-CoV-2 in HIV Infection: A Single-Arm Substudy of a Phase 2/3 Clinical Trial. Lancet HIV (2021) 8(8):e474-85. doi: 10.1016/S2352-3018(21)00103-X

4. Touizer E, Alrubayyi A, Rees-Spear C, Fisher-Pearson N, Griffith SA, Muir L, et al. Failure to Seroconvert After Two Doses of BNT162b2 SARS-CoV-2 Vaccine in a Patient With Uncontrolled HIV. Lancet HIV (2021) 8:e317-8. doi: 10.1016/S2352-3018(21)00099-0

5. Ruddy JA, Boyarsky BJ, Werbel WA, Bailey JR, Karaba AH, Garonzik-Wang JM, et al. Safety and Antibody Response to the First Dose of SARS-CoV-2 Messenger RNA Vaccine in Persons With HIV. AIDS (2021) 35(11):1872-4. doi: 10.1097/QAD.0000000000002945

6. Woldemeskel BA, Karaba AH, Garliss CC, Beck EJ, Wang KH, Laeyendecker O, et al. The BNT162b2 mRNA Vaccine Elicits Robust Humoral and Cellular Immune Responses in People Living With HIV. Clin Infect Dis (2021) 22: ciab648. doi: 10.1093/cid/ciab648

7. Clinical Features and Prognostic Factors of COVID-19 in People Living With HIV Hospitalized With Suspected or Confirmed SARS-CoV-2 Infection . Available at: https://www.who.int/publications-detail-redirect/WHO-2019nCoV-Clinical-HIV-2021.1 (Accessed August 18, 2021).

8. Johnston R. The First 6 Months of HIV-SARS-CoV-2 Coinfection: Outcomes for 6947 Individuals. Curr Opin HIV AIDS (2021) 16:54-62. doi: 10.1097/ COH.0000000000000654

9. Lerner AM, Eisinger RW, Fauci AS. Comorbidities in Persons With HIV: The Lingering Challenge. JAMA (2019) 323(1):19-20. doi: 10.1001/ jama.2019.19775

10. CDC. Science Brief: Evidence Used to Update the List of Underlying Medical Conditions That Increase a Person's Risk of Severe Illness From COVID-19 (2020). Centers for Disease Control and Prevention. Available at: https://www. cdc.gov/coronavirus/2019-ncov/hcp/clinical-care/underlying-evidence-table. html (Accessed April 13, 2021).

11. UNAIDS. Global HIV \& AIDS Statistics. Available at: https://www.unaids.org/ en/resources/fact-sheet (Accessed June 24, 2019).

12. Erasmus JH, Khandhar AP, O'Connor MA, Walls AC, Hemann EA, Murapa P, et al. An Alphavirus-Derived Replicon RNA Vaccine Induces SARS-CoV-2 contract 27220140006C (Erasmus). MO'C is also supported by $\mathrm{NIH}$ grant K01-MH123258.

\section{ACKNOWLEDGMENTS}

We thank the WaNPRC animal staff for the excellent care of the animals, to Dr. Brandon Keele for the SIVmac239M virus, the WaNPRC Virology and Immunology Core for viral loads and CBC subsets, the Department of Laboratory Medicine, and to C. Cannon, J. Shah, and A. Wald for feedback on the manuscript.

\section{SUPPLEMENTARY MATERIAL}

The Supplementary Material for this article can be found online at: https://www.frontiersin.org/articles/10.3389/fimmu.2021. 800723/full\#supplementary-material

Neutralizing Antibody and T Cell Responses in Mice and Nonhuman Primates. Sci Transl Med (2020) 12:eabc9396. doi: 10.1126/scitranslmed.abc9396

13. HDT Bio Corp and Gennova Complete Phase 1 Trial of COVID-19 RNA Vaccine in India. HDT Bio. Available at: https://www.hdt.bio/news-blog/hdtbio-corp-and-gennova-complete-phase-1-trial-of-covid-19-rna-vaccine-inindia (Accessed October 22, 2021).

14. HDT Bio Partners With Brazil's SENAI CIMATEC to Advance RNA COVID19 Vaccine to Fight Pandemic. HDT Bio. Available at: https://www.hdt.bio/ news-blog/hdt-bio-corp-and-senai-cimatec-to-develop-covid19-vaccine (Accessed October 22, 2021).

15. HDT Bio Announces Agreement With Korea's Quratis to Co-Develop Innovative COVID-19 Vaccine in Asia. HDT Bio. Available at: https://www hdt.bio/news-blog/hdt-bio-announces-agreement-with-koreas-quratis-to-codevelop-innovative-covid-19-vaccine-in-asia (Accessed October 22, 2021).

16. Crum-Cianflone NF, Iverson E, Defang G, Blair PJ, Eberly LE, Maguire J, et al. Durability of Antibody Responses After Receipt of the Monovalent 2009 Pandemic Influenza A (H1N1) Vaccine Among HIV-Infected and HIV-Uninfected Adults. Vaccine (2011) 29:3183-91. doi: 10.1016/j.vaccine.2011.02.040

17. Catherine F-X, Piroth L. Hepatitis B Virus Vaccination in HIV-Infected People: A Review. Hum Vaccin Immunother (2017) 13:1304-13. doi: 10.1080/ 21645515.2016.1277844

18. Chodick G, Tene L, Rotem RS, Patalon T, Gazit S, Ben-Tov A, et al. The Effectiveness of the TWO-DOSE BNT162b2 Vaccine: Analysis of Real-World Data. Clin Infect Dis (2021) 17:ciab438. doi: 10.1093/cid/ciab438

19. Khan N, Mahmud N. Effectiveness of SARS-CoV-2 Vaccination in a Veterans Affairs Cohort of Patients With Inflammatory Bowel Disease With Diverse Exposure to Immunosuppressive Medications. Gastroenterology (2021) 161:827-36. doi: 10.1053/j.gastro.2021.05.044

20. Tenforde MW, Patel MM, Ginde AA, Douin DJ, Talbot HK, Casey JD, et al. Effectiveness of SARS-CoV-2 mRNA Vaccines for Preventing Covid-19 Hospitalizations in the United States. Clin Infect Dis (2021) 6:ciab687. doi: 10.1101/2021.07.08.21259776

21. Vogel AB, Lambert L, Kinnear E, Busse D, Erbar S, Reuter KC, et al. SelfAmplifying RNA Vaccines Give Equivalent Protection Against Influenza to mRNA Vaccines But at Much Lower Doses. Mol Ther (2018) 26:446-55. doi: 10.1016/j.ymthe.2017.11.017

22. Fennessey CM, Pinkevych M, Immonen TT, Reynaldi A, Venturi V, Nadella P, et al. Genetically-Barcoded SIV Facilitates Enumeration of Rebound Variants and Estimation of Reactivation Rates in Nonhuman Primates Following Interruption of Suppressive Antiretroviral Therapy. PloS Pathog (2017) 13:e1006359. doi: 10.1371/journal.ppat.1006359

23. Chaplin JW, Chappell CP, Clark EA. Targeting Antigens to CD180 Rapidly Induces Antigen-Specific IgG, Affinity Maturation, and Immunological Memory. J Exp Med (2013) 210:2135-46. doi: 10.1084/jem.20130188 
24. Tunggal HC, Munson PV, O'Connor MA, Hajari N, Dross SE, Bratt D, et al. Effects of Therapeutic Vaccination on the Control of SIV in Rhesus Macaques With Variable Responsiveness to Antiretroviral Drugs. PloS One (2021) 16: e0253265. doi: 10.1371/journal.pone.0253265

25. Ho O, Larsen K, Polacino P, Li Y, Anderson D, Song R, et al. Pathogenic Infection of Macaca Nemestrina With a CCR5-Tropic Subtype-C Simian-Human Immunodeficiency Virus. Retrovirology (2009) 6:65. doi: 10.1186/1742-4690-6-65

26. Walls AC, Park Y-J, Tortorici MA, Wall A, McGuire AT, Veesler D. Structure, Function, and Antigenicity of the SARS-CoV-2 Spike Glycoprotein. Cell (2020) 181:281-92.e6. doi: 10.1016/j.cell.2020.02.058

27. Pepini T, Pulichino A-M, Carsillo T, Carlson AL, Sari-Sarraf F, Ramsauer K, et al. Induction of an IFN-Mediated Antiviral Response by a Self-Amplifying RNA Vaccine: Implications for Vaccine Design. J Immunol (2017) 198:401224. doi: 10.4049/jimmunol.1601877

28. Alrubayyi A, Gea-Mallorquí E, Touizer E, Hameiri-Bowen D, Kopycinski J, Charlton B, et al. Characterization of Humoral and SARS-CoV-2 Specific T Cell Responses in People Living With HIV. Nat Commun (2021) 12(1):5839. doi: 10.1101/2021.02.15.431215

29. Mascolo S, Romanelli A, Carleo MA, Esposito V. Could HIV Infection Alter the Clinical Course of SARS-CoV-2 Infection? When Less Is Better. J Med Virol (2020) 92:1777-8. doi: 10.1002/jmv.25881

30. Eisinger RW, Lerner AM, Fauci AS. Human Immunodeficiency Virus/AIDS in the Era of Coronavirus Disease 2019: A Juxtaposition of 2 Pandemics. J Infect Dis (2021) 224(9):1455-61. doi: 10.1093/infdis/jiab114

31. Shou S, Liu M, Yang Y, Kang N, Song Y, Tan D, et al. Animal Models for COVID-19: Hamsters, Mouse, Ferret, Mink, Tree Shrew, and Non-Human Primates. Front Microbiol (2021) 12:626553. doi: 10.3389/fmicb.2021.626553

32. Muñoz-Fontela C, Dowling WE, Funnell SGP, Gsell P-S, Riveros-Balta AX, Albrecht RA, et al. Animal Models for COVID-19. Nature (2020) 586:509-15. doi: 10.1038/s41586-020-2787-6

33. Melton A, Doyle-Meyers LA, Blair RV, Midkiff C, Melton HJ, RussellLodrigue $\mathrm{K}$, et al. The Pigtail Macaque (Macaca Nemestrina) Model of COVID-19 Reproduces Diverse Clinical Outcomes and Reveals New and Complex Signatures of Disease. bioRxiv (2021) 2021.08.28.458047. doi: 10.1101/2021.08.28.458047

34. Fahlberg MD, Blair RV, Doyle-Meyers LA, Midkiff CC, Zenere G, RussellLodrigue KE, et al. Cellular Events of Acute, Resolving or Progressive COVID19 in SARS-CoV-2 Infected non-Human Primates. Nat Commun (2020) 11:6078. doi: 10.1038/s41467-020-19967-4

35. Klatt NR, Canary LA, Vanderford TH, Vinton CL, Engram JC, Dunham RM, et al. Dynamics of Simian Immunodeficiency Virus SIVmac239 Infection in Pigtail Macaques. J Virol (2012) 86:1203-13. doi: 10.1128/JVI.06033-11

36. Canary LA, Vinton CL, Morcock DR, Pierce JB, Estes JD, Brenchley JM, et al. Rate of AIDS Progression is Associated With Gastrointestinal Dysfunction in Simian Immunodeficiency Virus-Infected Pigtail Macaques. J Immunol (2013) 190:2959-65. doi: 10.4049/jimmunol.1202319

37. Klatt NR, Harris LD, Vinton CL, Sung H, Briant JA, Tabb B, et al. Compromised Gastrointestinal Integrity in Pigtail Macaques is Associated With Increased Microbial Translocation, Immune Activation, and IL-17 Production in the Absence of SIV Infection. Mucosal Immunol (2010) 3:387-98. doi: 10.1038/mi.2010.14

38. Pandrea I, Landay A, Wilson C, Stock J, Tracy R, Apetrei C. Using the Pathogenic and Nonpathogenic Nonhuman Primate Model for Studying Non-AIDS Comorbidities. Curr HIV/AIDS Rep (2015) 12:54-67. doi: 10.1007/s11904-014-0245-5

39. Pandrea I, Cornell E, Wilson C, Ribeiro RM, Ma D, Kristoff J, et al. Coagulation Biomarkers Predict Disease Progression in SIV-Infected
Nonhuman Primates. Blood (2012) 120:1357-66. doi: 10.1182/blood-2012 03-414706

40. Blair RV, Vaccari M, Doyle-Meyers LA, Roy CJ, Russell-Lodrigue K, Fahlberg $\mathrm{M}$, et al. Acute Respiratory Distress in Aged, SARS-CoV-2-Infected African Green Monkeys But Not Rhesus Macaques. Am J Pathol (2021) 191:274-82. doi: 10.1016/j.ajpath.2020.10.016

41. Urano E, Okamura T, Ono C, Ueno S, Nagata S, Kamada H, et al. COVID19 Cynomolgus Macaque Model Reflecting Human COVID-19 Pathological Conditions. PNAS (2021) 118:e2104847118. doi: 10.1073/ pnas. 2104847118

42. Hasenkrug KJ, Feldmann F, Myers L, Santiago ML, Guo K, Barrett BS, et al. Recovery From Acute SARS-CoV-2 Infection and Development of Anamnestic Immune Responses in T Cell-Depleted Rhesus Macaques. mBio (2021) 12(4):e0150321. doi: 10.1128/mBio.01503-21

43. Kernéis S, Launay O, Turbelin C, Batteux F, Hanslik T, Boëlle P-Y. Long-Term Immune Responses to Vaccination in HIV-Infected Patients: A Systematic Review and Meta-Analysis. Clin Infect Dis (2014) 58:1130-9. doi: 10.1093/cid/ cit937

44. Spinelli MA, Lynch KL, Yun C, Glidden DV, Peluso MJ, Henrich TJ, et al. SARS-CoV-2 Seroprevalence, and IgG Concentration and Pseudovirus Neutralising Antibody Titres After Infection, Compared by HIV Status: A Matched Case-Control Observational Study. Lancet HIV (2021) 8:e334-41. doi: 10.1016/S2352-3018(21)00072-2

45. CDC. COVID-19 Vaccination (2021). Centers for Disease Control and Prevention. Available at: https://www.cdc.gov/coronavirus/2019-ncov/ vaccines/recommendations/immuno.html (Accessed August 17, 2021).

46. Choi B, Choudhary MC, Regan J, Sparks JA, Padera RF, Qiu X, et al. Persistence and Evolution of SARS-CoV-2 in an Immunocompromised Host. N Engl J Med (2020) 383:2291-3. doi: 10.1056/NEJMc2031364

47. Goldhill DH, Barclay WS. 2020 Hindsight - Should Evolutionary Virologists Have Expected the Unexpected During a Pandemic? Evolution (2021) 75 (9):2311-6. doi: 10.1111/evo.14317

Conflict of Interest: JE, JA, and DF have equity interest in HDT Bio. JE is a consultant for InBios. DF is a consultant for Gerson Lehrman Group, Orlance, Abacus Bioscience, Neoleukin Therapeutics. JE is a co-inventor on U.S. patent application no. 62/993,307 "Compositions and methods for delivery of RNA" pertaining to the LION formulation.

The remaining authors declare that the research was conducted in the absence of any commercial or financial relationships that could be construed as a potential conflict of interest.

Publisher's Note: All claims expressed in this article are solely those of the authors and do not necessarily represent those of their affiliated organizations, or those of the publisher, the editors and the reviewers. Any product that may be evaluated in this article, or claim that may be made by its manufacturer, is not guaranteed or endorsed by the publisher.

Copyright (c) 2021 O’Connor, Erasmus, Randall, Archer, Lewis, Brown, Fredericks, Groenier, Iwayama, Ahrens, Garrison, Wangari, Guerriero and Fuller. This is an open-access article distributed under the terms of the Creative Commons Attribution License (CC BY). The use, distribution or reproduction in other forums is permitted, provided the original author(s) and the copyright owner(s) are credited and that the original publication in this journal is cited, in accordance with accepted academic practice. No use, distribution or reproduction is permitted which does not comply with these terms. 